\title{
A multifrequency study of the active star forming region NGC 6357
}

\author{
C. E. Cappa ${ }^{1,2}$, R. H. Barbá ${ }^{3}$ M. Arnal ${ }^{1,2}$, N. Duronea ${ }^{2}$, \\ E. Fernández Lajús ${ }^{1}$, W. M. Goss ${ }^{4}$ and J. Vasquez ${ }^{2}$ \\ ${ }^{1}$ Facultad de Ciencias Astronómicas y Geofisicas, UNLP, Argentina \\ ${ }^{2}$ Instituto Argentino de Radioastronomía, Argentina \\ ${ }^{3}$ Departamento de Física, Universidad de La Serena, Chile \\ ${ }^{4} \mathrm{NRAO}$, Socorro, USA
}

To investigate the interaction of the massive stars with the gas and dust in the active star forming region NGC 6357, located in the Sagittarius spiral arm at a distance of 1.7-2.6 kpc (Massey et al. 2001), we analyzed the distribution of the neutral and ionized gas, and that of the dust, based on $\mathrm{H} \alpha$, [OIII] and [SII] images obtained with the CurtisSchmidt telescope at CTIO, radio continuum observations at $1.465 \mathrm{MHz}$ obtained with the Very Large Array (NRAO) in the DnC configuration (synthesized beam = 38"), HI data from the Parkes survey (angular resolution $\left.=15^{\prime}\right), \mathrm{CO}(1-0)$ observations obtained with the Nanten radiotelescope at Las Campanas Observatory (angular resolution = $2.7^{\prime}$ ), and IR images in the four MSX bands (angular resolution $=18.3^{\prime \prime}$ ).

NGC 6357 consists of a low excitation ionized envelope, 50' in diameter, HiI regions in different evolutionary stages (Felli et al. 1990), molecular clouds, OB stars (most of them belonging to the open cluster Pismis 24), and IR sources (Persi et al. 1986).

The $[\mathrm{SII}] / \mathrm{H} \alpha$ and $[\mathrm{OIII}] / \mathrm{H} \alpha$ line ratios confirm the low excitation of the ionized envelope. Although the Hi emission distribution is dominated by absorption due to radio continuum sources, the optical filaments to the $\mathrm{E}$ and $\mathrm{W}$ are seen surrounded by neutral gas with velocities in the range -12 to $+2 \mathrm{~km} \mathrm{~s}^{-1}$. The $\mathrm{CO}$ emission distribution shows molecular gas associated with the region with velocities in the range -7 to $+1 \mathrm{~km} \mathrm{~s}^{-1}$.

A detailed analysis of the region reveals interstellar bubbles and photodissociation regions created by the massive stars in Pi 24 and by undisclosed ionizing sources.

Keywords. ISM: HII regions - ISM: individual (NGC 6357) - ISM: bubbles

\section{Acknowledgements}

CEC would like to thank financial support from the IAU and the Check Academy of Sciences, which facilitated her participation in the Symposium. RHB acknowledges financial support from FONFECYT (Chile) Programs No. 1050052 and 7050215. This work was partially financed by CONICET of Argentina under project PIP 5886/05 and Universidad Nacional de La Plata, Argentina, under project 11/G072. The National Radio Astronomy Observatory is a facility of the National Science Foundation operated under cooperative agreement by Associated Universities, Inc.

\section{References}

Felli, M., Persi, P., Roth, M., et al. 1990, A\&A 232, 477

Massey, P., DeGioia-Eastwood, K. \& Waterhouse, E. 2001, AJ 121, 1050

Persi, P., Ferrari-Toniolo, M., Roth, M. \& Tapia, M. 1986, A\&\&A 170, 97 